The Quarterly Journal of Mathematics Advance Access published online on 23 May 2005

Quart. J. Math. 56 (2005), 431-442; doi:10.1093/qmath/hah041

\title{
TRACE FIELDS OF REPRESENTATIONS AND VIRTUALLY HAKEN 3-MANIFOLDS
}

\author{
by XINGRU ZHANG ${ }^{\dagger}$ \\ (Department of Mathematics, SUNY at Buffalo, Buffalo, NY 14260-2900, USA)
}

[Received 8 May 2004. Revised 15 September 2004]

\begin{abstract}
We introduce and study trace fields and invariant trace fields of $S L_{2}(\mathbb{C})$ and $P S L_{2}(\mathbb{C})$ representations of 3-manifold groups. We give conditions on such fields which imply that the underlying 3-manifold is virtually Haken or has virtually positive or $\infty$ first Betti number. In particular, we define the notion of an algebraically trace-proper surface subgroup in a 3-manifold group, and show that any closed orientable irreducible 3-manifold with such a surface subgroup is virtually Haken. We give infinitely many families of closed orientable hyperbolic non-Haken 3-manifolds with algebraically trace-proper surface subgroups.
\end{abstract}

\section{Introduction}

In this paper a 3-manifold is always assumed to be connected and orientable, without loss of generality for the problems to be considered. A 3-manifold is said to be Haken if it is compact, irreducible, and contains a properly embedded incompressible surface. A 3-manifold is said to be virtually Haken if it has a finite cover which is Haken. The well-known virtually Haken conjecture states that every closed and irreducible 3-manifold with infinite fundamental group is virtually Haken. It is also conjectured that every closed Haken 3-manifold has virtually positive first Betti number, that is, the manifold has a finite cover which has positive first Betti number. If $M$ is a closed hyperbolic Haken 3-manifold, then it is further conjectured that $M$ has virtually $\infty$ first Betti number, which means that for any positive integer $n$, there is a finite cover $M_{n}$ of $M$ such that the first Betti number of $M_{n}$ is larger than $n$. These conjectures are fundamental and difficult issues in 3-manifold topology. In this paper we provide some information about these conjectures by studying traces of representations of 3-manifold groups into $S L_{2}(\mathbb{C})$ or $P S L_{2}(\mathbb{C})$.

For a group $\Gamma$ and a representation $\rho: \Gamma \rightarrow S L_{2}(\mathbb{C})$, we define the trace field $K_{\rho}$ of $\rho$ to be the field generated by the traces of all the matrices $\rho(\gamma), \gamma \in \Gamma$, over the base field $\mathbb{Q}$ of rational numbers, that is,

$$
K_{\rho}=\mathbb{Q}(\operatorname{tr}(\rho(\gamma)) ; \quad \gamma \in \Gamma) .
$$

Recall that the character $\chi_{\rho}$ of $\rho$ is the complex-valued function $\chi_{\rho}: \Gamma \rightarrow \mathbb{C}$ defined by $\chi_{\rho}(\gamma)=\operatorname{tr}(\rho(\gamma))$ for $\gamma \in \Gamma$. Hence if two $S L_{2}(\mathbb{C})$-representations $\rho_{1}, \rho_{2}$ of $\Gamma$ have the same character, in particular if they are conjugate to each other, then $K_{\rho_{1}}=K_{\rho_{2}}$.

As in [17], for any group $\Gamma$ we use $\Gamma^{(2)}$ to denote the subgroup of $\Gamma$ generated by $\left\{\gamma^{2} ; \gamma \in \Gamma\right\}$. If $\Gamma$ is finitely generated, then $\Gamma^{(2)}$ is a finitely generated finite-index normal subgroup of $\Gamma$. For a representation $\rho: \Gamma \rightarrow S L_{2}(\mathbb{C})$, we use $\rho^{(2)}$ to denote the restriction

${ }^{\dagger}$ E-mail: xinzhang@math.buffalo.edu 
representation of $\rho$ on the subgroup $\Gamma^{(2)}$. We also use $K_{\rho}^{(2)}$ to denote the trace field of $\rho^{(2)}$, thus $K_{\rho}^{(2)}=K_{\rho(2)}$. We call $\mathrm{K}_{\rho}^{(2)}$ the invariant trace field of $\rho$.

Recall that a representation $\rho: \Gamma \rightarrow S L_{2}(\mathbb{C})$ is called reducible if $\rho(\Gamma)$, as a subgroup of $S L_{2}(\mathbb{C})$ acting on the complex vector space $\mathbb{C}^{2}$ in the standard way, has an invariant 1-dimensional subspace, and called irreducible otherwise. A representation $\rho: \Gamma \rightarrow S L_{2}(\mathbb{C})$ is called virtually reducible if there is a finite index subgroup $\Gamma_{1}$ of $\Gamma$ such that the restriction of $\rho$ on $\Gamma_{1}$ is a reducible representation, and called non-virtually reducible otherwise. Note that if $M$ is a hyperbolic 3-manifold of finite volume and if $\rho$ is a discrete faithful representation of $\pi_{1}(M)$ into $S L_{2}(\mathbb{C})$ then $\rho$ is non-virtually reducible.

We say that a 3-manifold $M$ has a surface subgroup if $\pi_{1}(M)$ contains a subgroup which is isomorphic to the fundamental group of a closed connected orientable surface of genus at least one. We call a surface subgroup $\Lambda$ of $\pi_{1}(M)$ algebraically trace-proper if $\pi_{1}(M)$ has a non-virtually reducible representation $\rho$ such that $K_{\rho \mid \Lambda}$ is a proper subfield of $K_{\rho}^{(2)}$, where $\left.\rho\right|_{\Lambda}$ denotes the restriction of $\rho$ on the subgroup $\Lambda$. Note that this notion is well associated to the conjugacy class of $\rho$ and to the conjugacy class of the subgroup $\Lambda$ in $\pi_{1}(M)$. The following theorem is the main result of this paper.

THEOREM 1.1 Any closed irreducible 3-manifold $M$ with an algebraically trace-proper surface subgroup is a virtually Haken 3-manifold.

If a closed hyperbolic 3-manifold $M$ has an immersed closed totally geodesic surface, then $M$ contains an algebraically trace-proper surface subgroup. In fact, let $\rho$ be a discrete faithful representation of $\pi_{1}(M)$ into $S L_{2}(\mathbb{C})$, then it is well known that $\rho\left(\Gamma^{(2)}\right)$ cannot be conjugated into $S L_{2}(\mathbb{R})$ or into $S U_{2}(\mathbb{C})$. Hence the character of $\rho^{(2)}$ is not a real-valued function by [14, Proposition III.1.1]. It follows that $K_{\rho}^{(2)}$ is a number field which is not contained in $\mathbb{R}$. On the other hand, if $\Lambda \subset \pi_{1}(M)$ is the subgroup corresponding to an immersion of a closed totally geodesic surface of $M$, then $\operatorname{tr}(\rho(\delta)) \in \mathbb{R}$ for every $\delta \in \Lambda$. Take $\Lambda_{1}$ a finite-index subgroup of $\Lambda^{(2)}$ corresponding to an immersion of a closed orientable surface; then $\operatorname{tr}(\rho(\delta)) \in \mathbb{R} \cap K_{\rho}^{(2)}=F$ for every $\delta \in \Lambda_{1}$ and $F$ is a proper subfield of $K_{\rho}^{(2)}$. That is, $\Lambda_{1}$ is an algebraically trace-proper surface subgroup of $\pi_{1}(M)$.

Corollary 1.2 [11] Any closed hyperbolic 3-manifold with an immersed closed totally geodesic surface is a virtually Haken 3-manifold.

We now give some conditions on the traces of an $S L_{2}(\mathbb{C})$-representation of a closed Haken 3 -manifold to ensure that the manifold has virtually positive or virtually $\infty$ first Betti number. Let $M$ be a closed Haken 3-manifold. Let $S$ be a connected, embedded, closed, incompressible surface in $M$. If $S$ separates $M$ into two parts $M_{1}$ and $M_{2}$, then $\pi_{1}(M)=\pi_{1}\left(M_{1}\right) * \pi_{1}(S) \pi_{1}\left(M_{2}\right)$ is a free product with amalgamation, and $\pi_{1}(S)$ can be considered as a subgroup of $\pi_{1}\left(M_{i}\right)$, and $\pi_{1}\left(M_{i}\right)$ can be considered as subgroup of $\pi_{1}(M)$, for each $i=1,2$. Here the base point for all the involved fundamental groups is in $S$. If $S$ does not separate $M$, then $\pi_{1}(M)=\pi_{1}\left(M_{1}\right) * \pi_{1(S)}$ is an HNN extension, where $M_{1}$ is $M \backslash S$, and $\pi_{1}(S)$ can be considered as a subgroup of $\pi_{1}\left(M_{1}\right)$, and $\pi_{1}\left(M_{1}\right)$ can be considered as a subgroup of $\pi_{1}(M)$. Here the base point for all the involved fundamental groups is in a parallel copy of $S$ in $M_{1}$. 
THeOREm 1.3 Let $M$ be a closed Haken 3-manifold and $S$ a connected, embedded, closed, orientable, incompressible surface in $M$.

(1) Suppose that $S$ separates $M$ into $M_{1}$ and $M_{2}$, and that there is an irreducible representation $\rho: \pi_{1}(M) \rightarrow S L_{2}(\mathbb{C})$ such that $K_{\rho}$ is a number field and $K_{\left.p\right|_{\pi_{1}(S)}}$ is a proper subfield of $K_{\left.p\right|_{\pi_{1}\left(M_{i}\right)}}$ for both $i=1,2$. Then $M$ has virtually positive first Betti number. If, in addition, $K_{\left.\rho\right|_{\pi_{1}\left(M_{i}\right)} ^{(2)}}$ is not contained in $K_{\left.p\right|_{\pi_{1}(S)}}$ for at least one of $i=1,2$, then $M$ has virtually $\infty$ first Betti number.

(2) Suppose that $S$ does not separate $M$ and that there is an irreducible representation $\rho: \pi_{1}(M) \rightarrow S L_{2}(\mathbb{C})$ such that $K_{\rho}$ is a number field and $K_{\left.\rho\right|_{\pi_{1}(S)}}$ is a proper subfield of $K_{\left.\rho\right|_{\pi_{1}\left(M_{1}\right)}}$, where $M_{1}=M \backslash S$. Then $M$ has virtually $\infty$ first Betti number.

If a closed hyperbolic 3-manifold $M$ contains an embedded closed totally geodesic surface $S$, then some finite cover of $M$ satisfies the conditions of (1) or (2) of Theorem 1.3. In fact, we may assume that $S$ is orientable (otherwise replace it by its orientable double cover). By passing to a double cover of $M$, we may further assume that $S$ is not the boundary of a twisted $I$-bundle in $M$. Let $\Lambda$ be the surface subgroup corresponding to $S$, and let $\rho$ be a discrete faithful representation of $\pi_{1}(M)$ into $S L_{2}(\mathbb{C})$. Then $K_{\rho \mid \Lambda}$ is a proper subfield of the number field $K_{\rho}$. If $S$ separates $M$ into $M_{1}$ and $M_{2}$, then each $M_{i}$ is not an $I$-bundle. Since $M_{i}$ has totally geodesic boundary and is not an $I$-bundle, a similar discussion as that following Theorem 1.1 shows that the character of $\left.\rho\right|_{\pi_{1}\left(M_{i}\right)} ^{(2)}$ cannot be a real-valued function. Therefore all the conditions of the part (1) of Theorem 1.3 are satisfied. If $S$ is non-separating, then $M_{1}=M \backslash S$ (completed with the path metric) is a 3-manifold with totally geodesic boundary. Similarly $M_{1}$ is not an $I$-bundle over a surface, and the trace field of $M_{1}$ is not contained in $\mathbb{R}$. Hence the conditions of the part (2) of Theorem 1.3 are satisfied. Therefore we have proved the following.

Corollary 1.4 [12] Any closed hyperbolic 3-manifold with an embedded closed totally geodesic surface has virtually $\infty$ first Betti number.

More generally we may consider the trace fields and invariant trace fields of $P S L_{2}(\mathbb{C})$ representations. For a subgroup $G$ of $S L_{2}(\mathbb{C})$, we define its trace field to be the field over $\mathbb{Q}$ generated by the traces of all the elements in $G$. Let $\Phi: S L_{2}(\mathbb{C}) \rightarrow P S L_{2}(\mathbb{C})$ be the canonical quotient homomorphism. For a group $\Gamma$ and a representation $\hat{\rho}: \Gamma \rightarrow P S L_{2}(\mathbb{C})$, we define the trace field $K_{\hat{\rho}}$ of $\hat{\rho}$ to be the trace field of the subgroup $\Phi^{-1}(\hat{\rho}(\Gamma))$ of $S L_{2}(\mathbb{C})$. Similarly, we use $\hat{\rho}^{(2)}$ to denote the restriction of $\hat{\rho}$ to the subgroup $\Gamma^{(2)}$ of $\Gamma$, and define the invariant trace of $\hat{\rho}$ to be the trace field of $\hat{\rho}^{(2)}$. A representation $\hat{\rho}: \Gamma \rightarrow P S L_{2}(\mathbb{C})$ is called reducible if $\Phi^{-1}(\hat{\rho}(\Gamma))$, as a subgroup of $S L_{2}(\mathbb{C})$, is reducible, and called irreducible otherwise. The representation $\hat{\rho}$ is virtually reducible if there is a finite-index subgroup $\Gamma_{1}$ of $\Gamma$ such that the restriction of $\hat{\rho}$ on $\Gamma_{1}$ is a reducible representation. It is easy to see that if $\rho: \Gamma \rightarrow S L_{2}(\mathbb{C})$ is an $S L_{2}(\mathbb{C})$-representation and $\hat{\rho}=\Phi \circ \rho$ is the corresponding $P S L_{2}(\mathbb{C})$-representation, then $K_{\rho}=K_{\hat{\rho}}$ and $K_{\rho(2)}=K_{\hat{\rho}(2)}$ (although $\rho(\Gamma)$ and $\Phi^{-1}(\hat{\rho}(\Gamma))$ might not be equal to each other), and $\rho$ is reducible or virtually reducible if and only if $\hat{\rho}$ is respectively. We shall also call a surface subgroup $\Lambda$ of a 3-manifold group $\pi_{1}(M)$ algebraically trace-proper if $\pi_{1}(M)$ has a non-virtually reducible $P S L_{2}(\mathbb{C})$ representation $\hat{\rho}$ such that $K_{\hat{\rho} \mid \Lambda}$ is a proper subfield of $K_{\hat{\rho}(2)}$.

THEOREM 1.5 Each of Theorem 1.1 and Theorem 1.3 still holds if the $S L_{2}(\mathbb{C})$-representation involved is replaced by a PSL $(\mathbb{C})$-representation with the corresponding property. 
The above theorems will be proved in section 2. Walter Neumann suggested an approach for constructing hyperbolic 3-manifolds with algebraically trace-proper but not necessarily totally geodesic surface subgroups and a similar method was suggested by Darren Long. The 3-manifolds they constructed are already Haken manifolds. In final section, we shall construct infinitely many families of non-Haken hyperbolic 3-manifolds with algebraically traceproper surface subgroups using a different method. We show there the following result.

THEOREM 1.6 Let $K$ be any ( $p, q, r)$-pretzel knot in $S^{3}$ with $p>0$ and $q>0$ odd, $r>0$ even, $\operatorname{gcd}(p, q)=d>1$. Then infinitely many surgeries on $K$ produce hyperbolic non-Haken 3-manifolds with algebraically trace-proper surface subgroups.

We do not know whether any of the non-Haken 3-manifolds given in Theorem 1.6 contains closed immersed totally geodesic surfaces.

It seems a strong condition on a 3-manifold to ask for it to have an algebraically trace-proper surface subgroup. It is not clear how big the class of irreducible 3-manifolds with algebraically trace-proper surface subgroups is. This class of 3-manifolds is probably much larger than the class of hyperbolic 3-manifolds with immersed totally geodesic surfaces, given the belief that every number field can be realized as the trace field of a hyperbolic 3-manifold, plus the freedom that in the definition of an algebraically trace-proper surface subgroup, the representation is not necessarily required to be discrete and faithful. It is not totally unreasonable to conjecture that every closed irreducible 3-manifold $M$ with infinite fundamental group virtually has algebraically trace-proper surface subgroups, that is, $M$ has a finite cover which has algebraically trace-proper surface subgroups. Of course this conjecture is stronger than the virtually Haken conjecture.

\section{Proofs of Theorems 1.1, 1.3 and 1.5}

From now on, $\Gamma$ denotes a finitely generated group. We use $R(\Gamma)$ to denote the set of all representations of $\Gamma$ into $S L_{2}(\mathbb{C})$, and $X(\Gamma)$ the corresponding set of characters. If $M$ is a path connected space with finitely generated fundamental group, we also use $R(M)$ and $X(M)$ to denote $R\left(\pi_{1}(M)\right.$ ) and $X\left(\pi_{1}(M)\right)$ respectively. Both $R(\Gamma)$ and $X(\Gamma)$ are complex affine algebraic sets, often called the $S L_{2}(\mathbb{C})$ representation variety and character variety of $\Gamma$ respectively.

For any compact 3-manifold $M$, if for some $\rho \in R(M)$, the trace field $K_{\rho}$ is not a number field, then by the Hilbert Nullstellensatz, $X(M)$ must be positive dimensional as an algebraic set, and thus in such a case $M$ contains a properly embedded essential surface by [5]. Therefore when $M$ is an irreducible non-Haken 3 -manifold, $K_{\rho}$ is always a number field for any representation $\rho \in R(M)$.

When $M$ is a hyperbolic 3-manifold of finite volume, its fundamental group $\pi_{1}(M)$ has a discrete faithful representation $\eta$ into $P S L_{2}(\mathbb{C})$. It is known that the $P S L_{2}(\mathbb{C})$-representation $\eta$ can be lifted to a $S L_{2}(\mathbb{C})$-representation $\rho$ [21]. An argument in [14] shows that $\eta$ has precisely, up to conjugation, $\left|H^{1}\left(M ; \mathbb{Z}_{2}\right)\right|$ such lifts. As any two such lifts of $\eta$ differ only by multiplying by an element of $H^{1}\left(M ; \mathbb{Z}_{2}\right)$ the trace field $K_{\rho}$ is uniquely associated to the conjugacy class of $\eta$. By the Mostow-Prasad rigidity there are exactly, up to conjugacy, two discrete faithful representations $\eta$ and $\bar{\eta}$ from $\pi_{1}(M)$ into $P S L_{2}(\mathbb{C})$, where $\bar{\eta}$ is obtained from $\eta$ by taking the complex conjugation, that is, if $\eta(\gamma)= \pm\left(\begin{array}{ll}a & b \\ c & d\end{array}\right)$ then $\bar{\eta}(\gamma)= \pm\left(\begin{array}{ll}\bar{a} & \bar{b} \\ \bar{c} & \bar{d}\end{array}\right)$. Let $\bar{\rho}$ be a lift of $\bar{\eta}$. We caution that in general $K_{\rho}$ may not always be equal to $K_{\bar{\rho}}$. Their precise relation is $K_{\bar{\rho}}=\bar{K}_{\rho}$. So the trace 
field of $M$ usually referred to in the literature is only well defined up to taking the complex conjugation.

Note also that if $M$ is a hyperbolic 3-manifold of finite volume and if $\rho \in R(M)$ is a discrete faithful representation then the trace field $K_{\rho}$ is always a number field, as follows from the Mostow-Prasad rigidity and the Hilbert Nullstelensatz.

For $\rho \in R(\Gamma)$, let

$$
A_{\rho}=\left\{\sum a_{i} \rho\left(\gamma_{i}\right) ; \quad a_{i} \in K_{\rho}, \quad \gamma_{i} \in \Gamma\right\}
$$

where all sums are finite.

Lemma 2.1 [1, Proposition 2.2] If $\rho \in R(\Gamma)$ is an irreducible representation, then $A_{\rho}$ is a quaternion algebra over $K_{\rho}$, that is, $A_{\rho}$ is a central, simple, and 4-dimensional algebra over $K_{\rho}$.

A pair of matrices $A$ and $B$ in $S L_{2}(\mathbb{C})$ is called a generic pair if $A$ and $B$ do not share an eigenvector and one of the two matrices has trace not equal to \pm 2 . If $\rho \in R(\Gamma)$ is irreducible, then $\rho(\Gamma)$ contains a generic pair. This follows from [5, Lemma 1.5.1]. If $\left(\rho\left(\alpha_{1}\right), \rho\left(\alpha_{2}\right)\right)$ form a generic pair, then an argument given in [9, p. 248] shows that as a $K_{\rho}$-vector space, $A_{\rho}$ has a basis of the form $\left\{I, \rho\left(\alpha_{1}\right), \rho\left(\alpha_{2}\right), \rho\left(\alpha_{1} \alpha_{2}\right)\right\}$, where $I$ is the identity matrix.

Lemma 2.2 Suppose that $\rho \in R(\Gamma)$ is non-virtually-reducible. Then $\rho(\Gamma)$ contains a generic pair of the form $\left(\rho\left(\alpha^{2}\right), \rho\left(\beta^{2}\right)\right)$ such that each of $\rho\left(\alpha^{2}\right), \rho\left(\beta^{2}\right)$ and $\rho\left(\alpha^{2} \beta^{2}\right)$ has infinite order, and that the trace of each of $\rho\left(\alpha^{2}\right)$ and $\rho\left(\beta^{2}\right)$ is not equal to \pm 2 .

Proof. By Selberg's lemma, $\rho(\Gamma)$ has a finite-index torsion free normal subgroup $H$. Then $\Gamma_{1}=\rho^{-1}(H)$ is a finite-index subgroup of $\Gamma$. Since $\rho$ is non-virtually reducible, the restriction of $\rho$ on $\Gamma_{1}$ is irreducible. Thus, as noted immediately above, $\rho\left(\Gamma_{1}\right)=H$ contains a generic pair $\left(\rho(\alpha), \rho\left(\alpha_{1}\right)\right)$ with $\operatorname{tr}(\rho(\alpha)) \neq \pm 2, \alpha, \alpha_{1} \in \Gamma_{1}$. So $\rho\left(\alpha^{2}\right)$ has infinite order. As $\operatorname{tr}(\rho(\alpha)) \neq \pm 2$ and $\operatorname{tr}(\rho(\alpha)) \neq 0$ (since $\rho(\alpha)$ has infinite order), $\operatorname{tr}\left(\rho\left(\alpha^{2}\right)\right)=(\operatorname{tr}(\rho(\alpha)))^{2}-2$ cannot be equal to \pm 2 . Now for the given element $\rho\left(\alpha^{2}\right)$ we apply [5, Lemma 1.5.1] to see that there is an element $\rho(\beta)$ in $H$ such that $\operatorname{tr}(\rho(\beta)) \neq \pm 2$ and that $\rho\left(\alpha^{2}\right)$ and $\rho(\beta)$ form a generic pair. So $\rho\left(\beta^{2}\right)$ is also of infinite order and has trace $\neq \pm 2$. Now $\rho\left(\alpha^{2}\right)$ and $\rho\left(\beta^{2}\right)$ also form a generic pair since $\rho(\beta)$ and $\rho\left(\beta^{2}\right)$ have the same invariant 1-dimensional subspaces. As $\rho\left(\alpha^{2} \beta^{2}\right) \in H$ is non-trivial, it has infinite order.

Lemma 2.3 Suppose that $\rho \in R(\Gamma)$ is non-virtually reducible. Then

$$
K_{\rho}^{(2)}=\mathbb{Q}\left(\operatorname{tr}\left(\rho\left(\gamma^{2}\right)\right) ; \gamma \in \Gamma\right)
$$

Proof. Obviously by definition $K_{\rho}^{(2)}=K_{\rho(2)}=\mathbb{Q}\left(\operatorname{tr}(\rho(\delta)) ; \delta \in \Gamma^{(2)}\right) \supset \mathbb{Q}\left(\operatorname{tr}\left(\rho\left(\gamma^{2}\right)\right) ; \gamma \in \Gamma\right)$. So we need to show that $K_{\rho(2)} \subset \mathbb{Q}\left(\operatorname{tr}\left(\rho\left(\gamma^{2}\right)\right) ; \gamma \in \Gamma\right)$. Note also that $\mathbb{Q}\left(\operatorname{tr}\left(\rho\left(\gamma^{2}\right)\right) ; \gamma \in \Gamma\right)=$ $\mathbb{Q}\left((\operatorname{tr}(\rho(\gamma)))^{2} ; \gamma \in \Gamma\right)$ as $\operatorname{tr}\left(\rho\left(\gamma^{2}\right)\right)=(\operatorname{tr}(\rho(\gamma)))^{2}-2$. Thus we only need to show that $K_{\rho(2)} \subset$ $\mathbb{Q}\left((\operatorname{tr}(\rho(\gamma)))^{2} ; \gamma \in \Gamma\right)$.

By Lemma 2.2, $\rho(\Gamma)$ contains a generic pair of the form $\left(\rho\left(\alpha^{2}\right), \rho\left(\beta^{2}\right)\right)$ such that the trace of each of $\rho\left(\alpha^{2}\right)$ and $\rho\left(\beta^{2}\right)$ is not equal to \pm 2 and that all $\rho\left(\alpha^{2}\right), \rho\left(\beta^{2}\right), \rho\left(\alpha^{2} \beta^{2}\right)$ have infinite order (in particular their traces are not equal to 0 ). Therefore $A_{\rho}$ has a basis of the form 
$\left\{I, \rho\left(\alpha^{2}\right), \rho\left(\beta^{2}\right), \rho\left(\alpha^{2} \beta^{2}\right)\right\}$ by the note following Lemma 2.1. Now one can follow exactly the arguments used in the proof of [20, Proposition 4] to show that the vector space $A_{0}$ spanned by $\left\{I, \rho\left(\alpha^{2}\right), \rho\left(\beta^{2}\right), \rho\left(\alpha^{2} \beta^{2}\right)\right\}$ over the field $\mathbb{Q}\left((\operatorname{tr}(\rho(\gamma)))^{2} ; \gamma \in \Gamma\right)$ is a quaternion algebra over $\mathbb{Q}\left((\operatorname{tr}(\rho(\gamma)))^{2} ; \gamma \in \Gamma\right)$, that $\rho\left(\Gamma^{(2)}\right)$ is contained in $A_{0}$, and that $\operatorname{tr}(D) \in \mathbb{Q}\left((\operatorname{tr}(\rho(\gamma)))^{2} ; \gamma \in \Gamma\right)$ for every element $D \in A_{0}$. It follows that $K_{\rho(2)} \subset \mathbb{Q}\left((\operatorname{tr}(\rho(\gamma)))^{2} ; \gamma \in \Gamma\right)$.

Note that if $M$ is a hyperbolic 3-manifold of finite volume and if $\rho \in R(M)$ is a discrete faithful representation, then $K_{\rho}^{(2)}$ is the usual invariant trace field $K_{M}^{(2)}$ of $M$ defined in [17]. It is well associated to $M$ up to taking the complex conjugation. It is proved in [17] that $K_{M}^{(2)}$ is an invariant of the commensurability class of $M$, that is, if $M$ and $M_{1}$ have a finite-sheeted common cover, then $K_{M}^{(2)}=K_{M_{1}}^{(2)}$. For a group $\Gamma, K_{\rho}^{(2)}$ has the following property.

Proposition 2.4 Suppose that $\rho \in R(\Gamma)$ is a non-virtually reducible representation. Let $\Gamma_{1}$ be any finite-index subgroup of $\Gamma$. Let $\rho_{1} \in R\left(\Gamma_{1}\right)$ be the restriction of $\rho$ on $\Gamma_{1}$. Then $\mathrm{K}_{\rho_{1}}^{(2)}=\mathrm{K}_{\rho}^{(2)}$.

Proof. We follow an idea used in [17], originating from [20]. Choose a finite-index subgroup $\Gamma_{2}$ of $\Gamma_{1}^{(2)}$ such that $\Gamma_{2}$ is a normal subgroup of $\Gamma$ (such subgroup exists). Let $\rho_{2} \in R\left(\Gamma_{2}\right)$ be the restriction of $\rho$ on $\Gamma_{2}$. By definition, we have $K_{\rho_{2}} \subset K_{\rho_{1}}^{(2)} \subset K_{\rho}^{(2)}$. So it suffices to show that $K_{\rho}^{(2)} \subset K_{\rho_{2}}$. By Lemma 2.3 we only need to show that $\operatorname{tr}\left(\rho\left(\gamma^{2}\right)\right) \in K_{\rho_{2}}$ for every $\gamma \in \Gamma$.

The element $\rho(\gamma)$ induces an automorphism $\phi_{\gamma}$ of the group $\rho_{2}\left(\Gamma_{2}\right)=\rho\left(\Gamma_{2}\right)$ defined by

$$
\rho_{2}(\alpha) \stackrel{\phi_{\gamma}}{\longrightarrow} \rho(\gamma) \rho_{2}(\alpha) \rho(\gamma)^{-1}=\rho_{2}\left(\gamma \alpha \gamma^{-1}\right) \quad \text { for any } \rho_{2}(\alpha) \in \rho_{2}\left(\Gamma_{2}\right)
$$

Note that $\rho_{2} \in R\left(\Gamma_{2}\right)$ is an irreducible representation since $\rho$ is non-virtually reducible. Thus $A_{\rho_{2}}$ is a quaternion algebra over $K_{\rho_{2}}$ by Lemma 2.1. The above automorphism $\phi_{\gamma}$ extends naturally to an automorphism, which we still denote by $\phi_{\gamma}$, of the algebra $A_{\rho_{2}}$. By the SkölemNoether theorem, which states that any automorphism of a finite-dimensional central simple algebra is an inner automorphism, there is an invertible element $D_{0}$ of $\mathrm{A}_{\rho_{2}}$ such that $\phi_{\gamma}(D)=D_{0} D D_{0}^{-1}$ for any $D \in A_{\rho_{2}}$, that is, $\rho(\gamma) D \rho(\gamma)^{-1}=D_{0} D D_{0}^{-1}$ for any $D \in A_{\rho_{2}}$. As $A_{\rho_{2}} \otimes_{K_{\rho_{2}}} \mathbb{C} \cong M_{2}(\mathbb{C})$, where $M_{2}(\mathbb{C})$ is the algebra over $C$ of all $2 \times 2$ matrices, $D_{0}^{-1} \rho(\gamma)$ commutes with all elements of $M_{2}(\mathbb{C})$ and thus is a non-zero scalar matrix, that is, $D_{0}^{-1} \rho(\gamma)=a I$, $a \in \mathbb{C}^{*}$, where $I$ is the identity matrix. Since $\operatorname{det}(\rho(\gamma))=1, a^{2}=1 / \operatorname{det}\left(D_{0}\right) \in K_{\rho_{2}}$. Hence $\operatorname{tr}\left(\rho\left(\gamma^{2}\right)\right)=\operatorname{tr}\left(a^{2} D_{0}^{2}\right)=a^{2} \operatorname{tr}\left(D_{0}^{2}\right)=a^{2}\left(\left(\operatorname{tr}\left(D_{0}\right)\right)^{2}-2 \operatorname{det}\left(D_{0}\right)\right) \in K_{\rho_{2}}$. The proof is complete.

Lemma 2.5 Suppose that $\rho \in R(\Gamma)$ is irreducible and $K_{\rho}$ is a number field. Then $K_{\rho}$ has a finite field extension $L$ such that $\rho(\Gamma)$ can be conjugated into $S L_{2}(L)$.

Proof. Since $\rho$ is irreducible, $\rho(\Gamma)$ contains a generic pair $(\rho(\alpha), \rho(\beta))$. Now we apply [13, Proposition 3.2] to see that the assertion of the lemma follows. In fact if $\operatorname{tr}(\rho(\alpha)) \neq \pm 2$ and $\lambda$ is an eigenvalue of $\rho(\alpha)$ (note that $\lambda$ must be an algebraic number since $\operatorname{tr}(\rho(\alpha))$ is), then we may take $L=K_{\rho}(\lambda)[9$, Lemma 1.6].

It is known that if $M$ is a hyperbolic 3-manifold of finite volume, then the trace field $K_{M}$ of $M$ is a $\mathbb{Z}_{2}^{m}$-extension of the invariant trace field $K_{M}^{(2)}$ of $M$ for some non-negative integer $m$ [15].

Proposition 2.6 If $\rho \in R(M)$ is non-virtually reducible, then $K_{\rho}$ is a $\mathbb{Z}_{2}^{m}$-extension of $K_{\rho}^{(2)}$ for some non-negative integer $m$. If $M$ is a $\mathbb{Z}_{2}$-homology 3 -sphere, then $K_{\rho}=K_{\rho}^{(2)}$ for any $\rho \in R(M)$. 
Proof. Set $\Gamma=\pi_{1}(M)$. Then $\Gamma / \Gamma^{(2)}$ is a finite abelian group of exponent 2. In particular if $M$ is a $\mathbb{Z}_{2}$-homology 3 -sphere, then $\Gamma=\Gamma^{(2)}$. The conclusions of the proposition follow from Lemma 2.3 and the definition of $K_{\rho}^{(2)}$.

Lemma 2.7 (Jaco [18, Corollary 2.3]) Let $M$ be an irreducible closed 3-manifold. Suppose that $\pi_{1}(M)$ has a surface subgroup which is contained in infinitely many distinct subgroups of finite index in $\pi_{1}(M)$. Then $M$ is virtually Haken.

For any field $J$ and any field automorphism $\sigma$ of $J, \sigma$ extends naturally to a group automorphism $\sigma^{*}: S L_{2}(J) \rightarrow S L_{2}(J)$ by

$$
\sigma^{*}\left(\left(\begin{array}{ll}
a & b \\
c & d
\end{array}\right)\right)=\left(\begin{array}{ll}
\sigma(a) & \sigma(b) \\
\sigma(c) & \sigma(d)
\end{array}\right) .
$$

If $\rho: \Gamma \rightarrow S L_{2}(J)$ is a group representation, then $\sigma^{*} \circ \rho$ is also a representation of $\Gamma$ into $S L_{2}(J)$. In general, $\sigma^{*} \circ \rho$ may not be conjugate to $\rho$.

We are now ready to prove Theorem 1.1. Set $\Gamma=\pi_{1}(M)$ and let $\Lambda \subset \Gamma$ be a surface subgroup which is algebraically trace-proper with respect to a non-virtually reducible representation $\rho \in R(M)$, that is, if we set $F=K_{\left.p\right|_{\Lambda}}$, then $F$ is a proper subfield of $K_{\rho}^{(2)}$. By the note given in the second paragraph of this section, we may assume that $K_{\rho}$ is a number field. By Lemma 2.5, we have a finite-degree field extension $L$ of $K_{\rho}$ such that $\rho(\Gamma)$ can be conjugated into $S L_{2}(L)$. Since $K_{\rho}$ (as well as each of $K_{\rho}^{(2)}$ and $F$ ) is invariant under replacing $\rho$ by a conjugate of $\rho$, we may assume that $\rho(\Gamma)$ is already contained in $S L_{2}(L)$ for the given $\rho$.

By Lemma 2.7, we only need to show that $\Lambda$ is contained in infinitely many distinct finite index subgroups of $\Gamma$. It is equivalent to show that if $\Gamma$ has a finite-index proper subgroup $\Gamma_{j}$ which contains $\Lambda$, then $\Gamma_{j}$ itself has a finite-index proper subgroup $\Gamma_{j+1}$ which contains $\Lambda$.

By Proposition 2.4, if $\rho_{j}$ is the restriction of $\rho$ on the finite-index subgroup $\Gamma_{j}$ of $\Gamma$, then $K_{\rho_{j}}^{(2)}=K_{\rho}^{(2)}$. Thus $K_{\rho_{j}}$ is not contained in the field $F$. So there is an element $\gamma_{*}$ of $\Gamma_{j}$ such that $\operatorname{tr}\left(\rho\left(\gamma_{*}\right)\right)$ is not contained in $F$. It is now enough to show that there is a homomorphism $h$ of $\Gamma_{j}$ into a finite group such that $h\left(\gamma_{*}\right)$ is not contained in $h(\Lambda)$. For $\Gamma_{j+1}=h^{-1}(h(\Lambda))$ will be a finite index proper subgroup of $\Gamma_{j}$ containing $\Lambda$.

To find such a homomorphism $h$, we extend an idea used in [11]. Let $J$ be the Galois closure of $L$ as a number field, that is, $J$ is the minimal normal extension of $\mathbb{Q}$ containing $L$. Since $\operatorname{tr}\left(\rho\left(\gamma_{*}\right)\right)$ is not in $F$, there is an element $\sigma$ of the Galois group $G=\operatorname{Aut}(J / F)$ such that $\sigma\left(\operatorname{tr}\left(\rho\left(\gamma_{*}\right)\right)\right)-\operatorname{tr}\left(\rho\left(\gamma_{*}\right)\right) \neq 0$. Suppose that $\Gamma_{j}$ is generated by elements $\gamma_{1}, \ldots, \gamma_{n}$. Then $\rho\left(\Gamma_{j}\right)$ is generated by $\rho\left(\gamma_{1}\right), \ldots, \rho\left(\gamma_{n}\right)$. Let $R$ be the subring of $J$ generated by all the entries of $\rho\left(\gamma_{1}\right), \ldots, \rho\left(\gamma_{n}\right)$, and all the images of these entries under the map $\sigma$, together with the identity element 1 . Then $R$ is a finitely generated integral domain with 1 , and both $\rho\left(\Gamma_{j}\right)$ and $\sigma^{*}\left(\rho\left(\Gamma_{j}\right)\right)$ are contained in $S L_{2}(R)$. For such $R$ the intersection of all the maximal ideals is the zero element and the quotient of $R$ by any maximal ideal is a finite field (see, for example, [22, 4.1]). Now since $\sigma\left(\operatorname{tr}\left(\rho\left(\gamma_{*}\right)\right)\right)-\operatorname{tr}\left(\rho\left(\gamma_{*}\right)\right) \neq 0$, there is a maximal ideal $I$ of $R$ which does not contain the element $\sigma\left(\operatorname{tr}\left(\rho\left(\gamma_{*}\right)\right)\right)-\operatorname{tr}\left(\rho\left(\gamma_{*}\right)\right)$. Let $\phi: S L_{2}(R) \rightarrow S L_{2}(R / I)$ be the canonical projection homomorphism and let $\psi: S L_{2}(R) \rightarrow S L_{2}(R / I) \times S L_{2}(R / I)$ be the map defined by sending a matrix $D$ of $S L_{2}(R)$ to $\left(\phi(D), \phi\left(\sigma^{*}(D)\right)\right)$. Then $\psi$ is a well-defined group homomorphism. The composition $h=\psi \circ \rho$ is the required homomorphism from $\Gamma_{j}$ to the finite group $S L_{2}(R / I) \times S L_{2}(R / I)$. In fact, 
for any element $\delta$ of $\Lambda, h(\delta)$ is a pair of matrices with the same trace (since $\sigma$ fixes $F$ pointwise), while $h\left(\gamma_{*}\right)$ is a pair of matrices with distinct traces. The proof of Theorem 1.1 is now complete.

Now we prove part (1) of Theorem 1.3. Set $\Gamma=\pi_{1}(M), \Omega_{i}=\pi_{1}\left(M_{i}\right)$ and $\Lambda=\pi_{1}(S)$. Then $\Gamma=\Omega_{1} *_{\Lambda} \Omega_{2}$ is a free product with amalgamation. We apply the following lemma from [12]. Recall that a group $\Gamma$ is said to have virtually free quotient if $\Gamma$ has a finite-index subgroup which has a homomorphism onto a non-abelian free group. Note that virtually free quotient implies virtually $\infty$ first Betti number.

Lemma 2.8 [12, Lemma 2.2] (a) Assume that the group $\Gamma=\Omega_{1^{*} \Lambda} \Omega_{2}$ has a finite quotient $q: \Gamma \rightarrow G$ such that $q(\Lambda)$ is a proper subgroup of $q\left(\Omega_{i}\right)$ for both $i=1,2$; then $\Gamma$ has virtually positive first Betti number.

(b) If, in addition to (a), the index of $q(\Lambda)$ in $q\left(\Omega_{i}\right)$ is larger than 2 for at least one of $i=1,2$, then $\Gamma$ has virtually free quotient.

So to prove the first assertion of part (1) of Theorem 1.3, we only need to provide a finite quotient of $\Gamma$ satisfying the conditions of part (a) of Lemma 2.8. As $K_{\rho \mid \Lambda}$ is a proper subfield of $K_{\rho \mid \Omega_{i}}$, there is an element $\gamma_{i} \in \Omega_{i}$, for each $i=1,2$, such that $\operatorname{tr}\left(\rho\left(\gamma_{i}\right)\right)$ is not contained in $K_{\rho \mid \Lambda}$. The proof of 1.1 implies that has a finite-index subgroup $\Gamma_{i}$ such that $\Gamma_{i}$ contains $\Lambda$ but does not contain $\gamma_{i}$. So $\Gamma_{3}=\Gamma_{1} \cap \Gamma_{2}$ is a finite-index subgroup of $\Gamma$ which contains $\Lambda$ but contains neither $\gamma_{1}$ nor $\gamma_{2}$. Let $\Gamma_{4}$ be the intersection of all the conjugates of $\Gamma_{3}$ in $\Gamma$. Then $\Gamma_{4}$ is a finiteindex normal subgroup of $\Gamma$ and $q: \Gamma \rightarrow \Gamma / \Gamma_{4}$ is the finite quotient sought as $q(\Lambda) \neq q\left(\Omega_{i}\right)$ because $q(\Lambda) \subset q\left(\Gamma_{3}\right)$ but $q\left(\gamma_{i}\right) \notin q\left(\Gamma_{3}\right)$, for each $i=1,2$.

Similarly to prove the second assertion of part (1) of Theorem 1.3 we use the additional hypothesis to find a finite quotient of $\Gamma$ as described in part (b) of Lemma 2.8. By that hypothesis there is $\gamma_{i} \in \Omega_{i}$ such that $\operatorname{tr}\left(\gamma_{i}^{2}\right)$ is not contained in $K_{\rho \mid \Lambda}$ for at least one of $i=1$, 2, say $i=1$ (applying Lemma 2.3). Again we can find a finite index subgroup $\Gamma_{1}$ of $\Gamma$ which contains $\Lambda$ but does not contain $\gamma_{1}^{2}$. As above one constructs $\Gamma_{2}, \Gamma_{3}=\Gamma_{1} \cap \Gamma_{2}, \Gamma_{4}$, and $q: \Gamma \rightarrow \Gamma / \Gamma_{4}$. But now the index of $q(\Lambda)$ in $q\left(\Omega_{1}\right)$ is larger than 2 since $q\left(\gamma_{1}^{2}\right)$ is not contained in $q\left(\Gamma_{3}\right)$ and part (b) of Lemma 2.8 may be applied to complete the proof.

The proof for part (2) of Theorem 1.3 is similar to that of part (1) but applying another lemma of [12] recorded below.

Lemma 2.9 [12, Lemma 2.4] Let $\Gamma=\Omega_{1 * \Lambda}$ be an HNN-extension. Suppose that there is a finite quotient $q: \Gamma \rightarrow G$ such that $q(\Lambda)$ is not contained in $q\left(\Omega_{1}\right)$. Then $\Gamma$ has virtually free quotient.

Finally we prove Theorem 1.5, that is, we prove Theorem 1.1 and Theorem 1.3 in the $P S L_{2}(\mathbb{C})$ setting. To prove the $P S L_{2}(\mathbb{C})$-counterpart of Theorem 1.1 , let $M$ be a closed irreducible 3-manifold with a non-virtually reducible $P S L_{2}(\mathbb{C})$ representation $\hat{\rho}$ and with a surface subgroup $\Lambda$ such that $K_{\hat{\rho} \mid \Lambda}$ is a proper subfield of $K_{\hat{\rho}^{(2)}}$. Set $\Gamma=\pi_{1}(M)$ and consider the subgroup $\Phi^{-1}(\hat{\rho}(\Gamma))$ of $S L_{2}(\mathbb{C})$. By definition $K_{\hat{\rho}}$ is the trace field of $\Phi^{-1}(\hat{\rho}(\Gamma))$. The group $\Phi^{-1}\left(\hat{\rho}\left(\Gamma^{(2)}\right)\right)$ is generated by $\left(\Phi^{-1}(\hat{\rho}(\Gamma))\right)^{(2)}$ and $-I$, where $I$ is the identity matrix. Hence the trace field of $\left(\Phi^{-1}(\hat{\rho}(\Gamma))\right)^{(2)}$ is equal to the trace field of $\Phi^{-1}\left(\hat{\rho}\left(\Gamma^{(2)}\right)\right)$. Now if $\Gamma_{1}$ is a finite-index subgroup of $\Gamma$, then $\Phi^{-1}\left(\hat{\rho}\left(\Gamma_{1}\right)\right)$ is a finite-index subgroup of $\left(\Phi^{-1}(\hat{\rho}(\Gamma))\right)^{(2)}$. By Proposition 2.4 the trace field of $\left(\Phi^{-1}\left(\hat{\rho}\left(\Gamma_{1}\right)\right)\right)^{(2)}$ is the same as that of $\left(\Phi^{-1}(\hat{\rho}(\Gamma))\right)$; It follows that $K_{\hat{\rho}_{1}^{(2)}}=K_{\hat{\rho}^{(2)}}$, that is, Proposition 2.4 holds for $P S L_{2}(\mathbb{C})$-representations as well.

Again by the Hilbert Nullstellensatz and [5] (cf. [3]) we may assume that $K_{\hat{\rho}}$ is a number field. By Lemma 2.5, we have a finite-degree field extension $L$ of $K_{\hat{\rho}}$ such that $\Phi^{-1}(\hat{\rho}(\Gamma))$ can be 
conjugated into $S L_{2}(L)$. Since $K_{\hat{\rho}}$ (as well as each of $K_{\hat{\rho}^{(2)}}$ and $K_{\hat{\rho} \mid \Lambda}$ ) is invariant under replacing $\hat{\rho}$ by a conjugate of $\hat{\rho}$, we may assume that $\Phi^{-1}(\hat{\rho}(\Gamma))$ is already contained in $S L_{2}(L)$ for the given $\hat{\rho}$. By Lemma 2.7, we only need to show that $\Lambda$ is contained in infinitely many distinct finite-index subgroups of $\Gamma$. This is equivalent to showing that the group $\Phi^{-1}(\hat{\rho}(\Lambda))$ is contained in infinitely many distinct finite-index subgroups of the group $\Phi^{-1}(\hat{\rho}(\Gamma))$. The proof that the latter holds now goes exactly as the part of proof of Theorem 1.1 given after Lemma 2.7, considering $\Phi^{-1}(\hat{\rho}(\Lambda))$ and $\Phi^{-1}(\hat{\rho}(\Gamma))$ as subgroups of $S L_{2}(J)$.

Theorem 1.3 can be similarly generalized to the $P S L_{2}(\mathbb{C})$-setting. We omit the obvious details.

\section{Non-Haken 3-manifolds with algebraically trace-proper surfaces}

In this section we construct many closed orientable hyperbolic non-Haken 3-manifolds with algebraically trace-proper surface subgroups.

Let $M$ be a hyperbolic knot manifold, that is, $M$ is a compact 3-manifold whose boundary is a torus and whose interior admits a complete hyperbolic metric of finite volume. We use $M(\alpha)$ to denote the Dehn filling of $M$ with slope $\alpha$ and use $\Delta(\alpha, \beta)$ to denote the distance between two slopes $\alpha$ and $\beta$ on $\partial M$.

Let $(F, \partial F) \subset(M, \partial M)$ be a connected compact orientable properly embedded incompressible and $\partial$-incompressible surface with non-empty boundary such that $F$ separates $M$. Let $b$ be the number of components of $\partial F$ and $g$ the genus of $F$. Since $F$ separates $M, b$ is even. Let $\beta_{1}, \ldots, \beta_{b}$ be the boundary components of $F$ indexed so that they appear successively on $\partial M$, and let $\beta$ be the slope represented by each $\beta_{i}$. Let $X_{1}$ and $X_{2}$ be the two components into which $F$ separates $M$.

Let $S_{k}$ be the closed immersed surface in $M$ constructed from $F$ by Freedman-Freedman tubing; that is, connecting each pair $\left\{\beta_{2 i-1}, \beta_{2 i}\right\}$ of boundary components of $F$ by an immersed annulus $A_{i, k}$ which winds around $\partial M$ (within a regular neighbourhood of $\partial M$ ) $k$ times, starting at the $X_{1}$-side. See Fig. 1 for an illustration when $b=2$ and $k=3$. It is shown in [4] that $S_{k}$ is an incompressible closed surface in $M$ and remains incompressible in $M(\alpha)$ if $k$ is sufficiently large and if the distance $\Delta(\alpha, \beta)$ between the slopes $\alpha$ and $\beta$ is sufficiently large. Explicit lower bounds for $k$ and for $\Delta(\alpha, \beta)$ are given in [10]. It is shown in [10] that if $k \geq P(F)+1$, then $S_{k}$ is $\pi_{1}$-injective in $M$, where $P(F)=6 g+4 b-6$, and that if $k \geq(3 P(F)+1) / 2$ and $\Delta(\alpha, \beta) \geq 15 P(F)+3$, then $S_{k}$ remains $\pi_{1}$-injective in $M(\alpha)$. A better estimate for $P(F)$ is

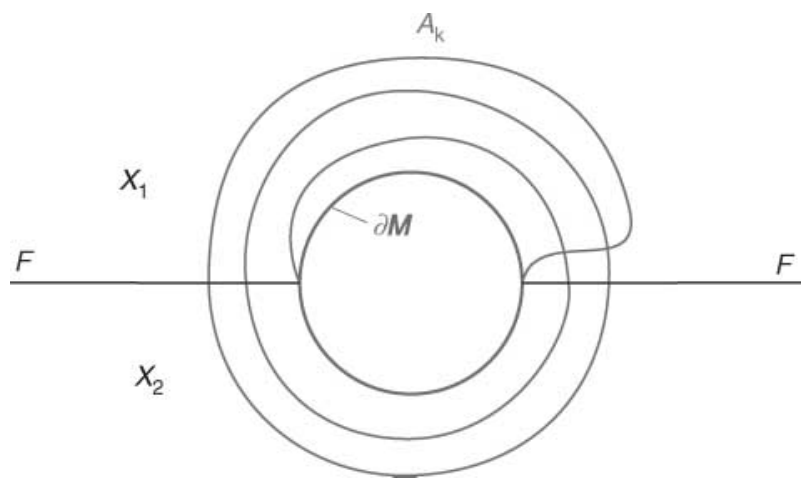

Fig. 1 The immersed closed surface $S_{k}=F \cup A_{k}$ in $M$ (when $b=2$ and $k=3$ ). 
independently obtained in [2], which is $4 g+3 b-3$. (That is, [10, Lemma 2.3] can be replaced by [2, Theorem 6.2.1].) We summarize this discussion as follows.

Lemмa 3.1 The closed immersed surface $S_{k}$ is $\pi_{1}$-injective in $M(\alpha)$ if $k \geq 6 g+\frac{9}{2} b-4$ and if $\Delta(\alpha, \beta) \geq 60 g+45 b-42$.

Let $\xi$ be a primitive $n$th root of unity, where $n>2$. It is well known that the extension degree of the field $\mathbb{Q}(\xi)$ over $\mathbb{Q}$ is equal to $\varphi(n)$, where $\varphi$ is the Euler function, and the degree of the field $\mathbb{Q}\left(\xi+\xi^{-1}\right)$ is $\varphi(n) / 2$. For simplicity, we just take a special case of this as given in the following lemma. Note that if $p$ is a prime factor of $n$, then $\varphi(n) \geq \varphi(p)=p-1$.

LEMMA 3.2 Let $\xi$ be a primitive nth root of unity such that $n$ contains a prime factor $p>2$. Then $[\mathbb{Q}(\xi), \mathbb{Q}] \geq p-1$ and $\left[\mathbb{Q}\left(\xi+\xi^{-1}\right), \mathbb{Q}\right] \geq(p-1) / 2$.

Let $G$ be a triangle group with the presentation $G=\left\langle x, y ; x^{m}=y^{n}=(x y)^{k}\right\rangle$. If $\frac{1}{m}+\frac{1}{n}+\frac{1}{k}<1$, the triangle group is hyperbolic, it is thus orbifold fundamental group of the hyperbolic 2-orbifold which is a 2 -sphere with three cone points of indices $m, n, k$. It is well known that any triangle group can be embedded in $P S L_{2}(\mathbb{C})$ as a discrete subgroup. So we may and will consider $G$ as a discrete subgroup of $P S L_{2}(\mathbb{C})$. If $G$ is hyperbolic, then it is a non-virtually reducible subgroup of $P S L_{2}(\mathbb{C})$.

Suppose $\Gamma$ is a group with a representation $\hat{\rho}: \Gamma \rightarrow P S L_{2}(\mathbb{C})$ whose image is a hyperbolic triangle group $G$ with a presentation as given above. Then $\hat{\rho}$ is non-virtually reducible. The trace field of $\hat{\rho}$ is generated by the traces of three fixed elements in $S L_{2}(\mathbb{C})$ with orders $2 m, 2 n$ and $2 k$ respectively. The trace of a matrix in $S L_{2}(\mathbb{C})$ of order $2 k$ is $\xi+\xi^{-1}$, where $\xi$ is a $2 k$ th root of unity. Hence, by Lemma 3.2, if $k$ is a prime number larger than 4 then $\left[K_{\hat{\rho}}: \mathbb{Q}\right] \geq \frac{1}{2}(k-1)>1$.

Let $K$ be a $(p, q, r)$ pretzel knot in $S^{3}$, where $p, q, r$ are all positive integers larger than one such that $r$ is an even integer, $p$ and $q$ are odd integers with their greatest common divisor $d=\operatorname{gcd}(p, q) \geq 3$. By [16], $K$ is a small knot, that is, its exterior $M$ in $S^{3}$ does not contain closed orientable incompressible non-boundary parallel surfaces. Hence by [7] all but finitely many Dehn surgeries on $K$ produce non-Haken 3-manifolds. By [21] all but finitely many surgeries on $K$ produce hyperbolic manifolds. By [8] (or by [6, Lemma 2.1] since $K$ is an alternating knot), the natural spanning surface (see Fig. 2(a) for an illustration) is a $\pi_{1}$-injective incompressible non-orientable surface (a punctured Klein bottle) in $M$ with boundary slope $2(p+q)$ (with respect to the standard meridian-longitude basis of $K$ ). The orientable double cover of this spanning surface in $M$ is an incompressible twice punctured torus. In fact, $K$ embeds in the boundary surface of a standard genus-two handlebody $H_{1}$ in $S_{3}$ such that $F=\partial H \backslash \stackrel{N}{(K}(K)$ is the incompressible twice punctured torus. Let $H_{2}$ be the other genus-two handlebody separated by $\partial H_{1}$ in $S^{3}$. The knot exterior $M$ is equal to $X_{1} \cup_{F} X_{2}$, where each $X_{i}$ is a genus-two handlebody. Note that $X_{1}$ is a regular neighbourhood of the spanning surface of $K$ mentioned above. (Fig. 2 illustrates the situation for the $(3,3,2)$-pretzel knot.) Let $\beta_{1}$ and $\beta_{2}$ be the two boundary components of $F$. Fix a point $x_{0}$ in $\beta_{1}$ as a base point for all the fundamental groups we shall be considering. Note that $K$ is a hyperbolic knot since $K$ is small and since any torus knot cannot have an embedded incompressible twice punctured torus in its exterior.

It is proved in [19] that $H_{1}\left(X_{1}, F\right)=\mathbb{Z}_{2}$ and $H_{1}\left(X_{2}, F\right)=\mathbb{Z}_{d}$, where $d=\operatorname{gcd}(p, q)$. It follows that there is a surjective homomorphism $\phi$ from $\pi_{1}(M)=\pi_{1}\left(X_{1}\right)_{* \pi_{1}(F)} \pi_{1}\left(X_{2}\right)$ to $\mathbb{Z}_{2} * \mathbb{Z}_{d}$ with $\phi\left(\pi_{1}\left(X_{1}\right)\right)=\mathbb{Z}_{2}, \phi\left(\pi_{1}\left(X_{2}\right)\right)=\mathbb{Z}_{d}$ and $\phi\left(\pi_{1}(F)\right)=1$. Let $\mu$ an element in $\pi_{1}(\partial M)$ represented by an oriented meridian of $K$ and let $\beta \in \pi_{1}(\partial M)$ be an element represented by the boundary component $\beta_{1}$ of $F$ with an orientation. Then $\mu$ and $\beta$ form a basis for $\pi_{1}(\partial M)$. From Fig. 2(b), 


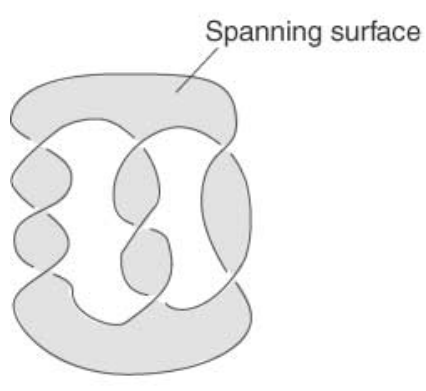

(a)

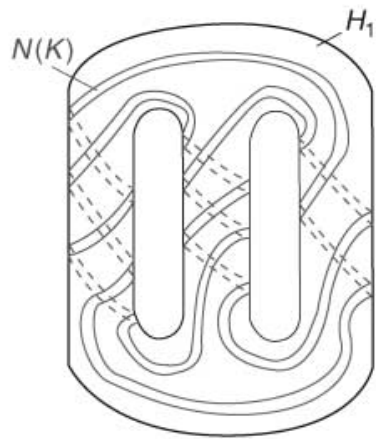

(b)

Fig. 2 (a) The spanning surface for a $(p, q, r)$-pretzel knot; (b) a $(p, q, r)$-pretzel knot in the boundary of a standard genus-two handlebody. (When $p=3, q=3$ and $r=2$.)

we see that $\mu$ can be expressed as $\gamma_{1} \gamma_{2}$ for some $\gamma_{1} \in \pi_{1}\left(X_{1}\right)$ and some $\gamma_{2} \in \pi_{1}\left(X_{2}\right)$. Since $\mu$ normally generates $\pi_{1}(M)$, it follows that $\phi(\mu)=\phi\left(\gamma_{1}\right) \phi\left(\gamma_{2}\right)=x y$, where $x$ is a generator of $\mathbb{Z}_{2}$ and $y$ is a generator of $\mathbb{Z}_{d}$. For any positive integer $k, \pi_{1}(M)$ has a representation $\hat{\rho}$ into $P S L_{2}(\mathbb{C})$ whose image is a triangle group of the form $\left\langle x, y ; x^{2}=y^{d}=(x y)^{k}=1\right\rangle$. Furthermore if $\alpha$ is a slope on $\partial M$ represented by $\mu^{k m} \beta^{n}$, then $\hat{\rho}$ factors through $\pi_{1}(M(\alpha))$; that is, $\hat{\rho}$ can be considered as a representation of $\pi_{1}(M(\alpha))$.

Now using this surface $F$, we construct a closed immersed surface $S_{k}$ in $M$ by FreedmanFreedman tubing as described earlier in this section. By Lemma 3.1, $S_{k}$ is $\pi_{1}$-injective in $M(\alpha)$ if $k \geq 11$ and if $\Delta(\alpha, \beta) \geq 108$. Now choose $k$ to be any fixed prime integer larger than 11 . From Fig. 1 and Fig. 2(b), it is easy to see that $\pi_{1}\left(S_{k+1}\right)$ is generated by $\pi_{1}(F)$ and $\mu^{k} \delta$ for some element $\delta \in \pi_{1}\left(X_{1}\right)$. Hence the image of $\pi_{1}\left(S_{k+1}\right)$ under the representation $\hat{\rho}$ is generated by $\hat{\rho}\left(\mu^{k} \delta\right)=\hat{\rho}(\delta)$. Thus the trace field $K_{\left.\hat{\rho}\right|_{\pi_{1}\left(S_{k+1}\right)}}$ is generated by the traces of elements in $\Phi^{-1}(\hat{\rho}(\delta))$ over $\mathbb{Q}$. But elements in $\Phi^{-1}(\hat{\rho}(\delta))$ are of order at most 4 . Hence their traces are in $\mathbb{Q}$. Therefore, $K_{\left.\hat{\rho}\right|_{\pi_{1}\left(S_{k+1}\right)}}=\mathbb{Q}$.

Let $\alpha$ be the slope represented by $\mu^{k m} \beta^{n}$ with $(k m, n)=1$. Then $\Delta(\alpha, \beta)=k m$. So if $m \geq 10$, then $S_{k+1}$ is $\pi_{1}$-injective in $M(\alpha)$. Also $\pi_{1}(M(\alpha))$ has the representation $\hat{\rho}$ whose image is the triangle group $\left\langle x, y ; x^{2}=y^{d}=(x y)^{k}\right\rangle$. As $k>10$, the triangle group is hyperbolic and thus $\hat{\rho}$ is non-virtually reducible. If $m$ is odd, then the first homology of $M(\alpha)$ is cyclic of odd order. Thus if we set $\Gamma=\pi_{1}(M(\alpha))$, then $\Gamma^{(2)}=\Gamma$. Therefore the invariant trace field of $\hat{\rho}$ (as a representation of $\Gamma$ ) is equal to the trace field of $\hat{\rho}$, whose extension degree is at least $\frac{1}{2}(k-1)$ by the discussion following Lemma 3.2.

In summary we have shown that for any slope $\alpha$ represented by $\mu^{m k} \beta^{n}$ with $(m k, n)=1$, $k \geq 11$ prime, $m \geq 10$ odd, $\pi_{1}(M(\alpha))$ has an algebraically trace-proper surface subgroup. Theorem 1.6 follows.

\section{Acknowledgements}

This research was partially supported by NSF grant DMS 0204428.

The author would like to thank Walter Neumann and Darren Long for helpful correspondence and conversations. 


\section{References}

1. H. Bass, Groups of integral representation type, Pacific J. Math. 86 (1980), 15-51.

2. S. Boyer, M. Culler, P. Shalen, and X. Zhang, Characteristic subsurfaces and Dehn filling, Trans. Amer. Math. Soc. to appear.

3. S. Boyer and X. Zhang, On Culler-Shalen seminorms and Dehn filling, Ann. of Math. 248 (1998), 737-801.

4. D. Cooper and D. Long, Some surface subgroups survive surgery, Geom. Topol. 5 (2001), $347-367$.

5. M. Culler and P. Shalen, Varieties of group representations and splittings of 3-manifolds, Ann. of Math. 117 (1983), 109-146.

6. C. Delman and R. Roberts, Alternating knots satisfy strong property P, Comment. Math. Helv. 74 (1999), 376-396.

7. A. Hatcher, On the boundary curves of incompressible surfaces, Pecific J. Math. 99 (1982), $373-377$.

8. A. Hatcher and U. Oertel, Boundary slopes for Montesinos knots, Topology 28 (1989), $453-480$.

9. H. Hilden, T. Lozano, and J. Montesinos, A characterization of arithmetic subgroups of SL(2, R) and SL(2, C), Math. Nachr. 159 (1992), 245-270.

10. T. Li, Immersed essential surfaces in hyperbolic 3-manifolds, Comm. Anal. Geom. 10 (2002), 275-290.

11. D. Long, Immersions and embeddings of totally geodesic surfaces, Bull. London Math. Soc. 19 (1987), 481-484.

12. A. Lubotzky, Free quotients and the first Betti number of some hyperbolic manifolds, Translation Groups 1 (1996), 71-82.

13. A. Macbeath, Commensurability of co-compact three-dimensional hyperbolic groups, Duke Math. J. 50 (1983), 1245-1253.

14. J. Morgan and P. Shalen, Valuations, trees, and degenerations of hyperbolic structures. I, Ann. of Math (2) 120, (1984), 401-476.

15. W. Neumann and A. Reid, Arithmetic of hyperbolic manifolds, Topology '90, (Columbus, OH, 1990), Ohio State Univ. Math. Res. Inst. Publ., 1, de Gruyter, Berlin, 1992, 273-310.

16. U. Oertel, Closed incompressible surfaces in complements of star links, Pacific J. Math. 111 (1984), 209-230.

17. A. Reid, A note on trace-fields of Kleinian groups, Bull. London Math. Soc. 22 (1990), $349-352$.

18. P. Shalen and P. Wagreich, Growth rates, $Z_{\mathrm{p}}$-homology, and volumes of hyperbolic 3-manifolds, Trans. Amer. Math. Soc. 331 (1992), 895-917.

19. J. Simon, Methods for proving that certain classes of knots have property P, Ph.D. Thesis, University of Wisconsin, 1969.

20. K. Takeuchi, A characterisation of arithmetic Fuchsian subgroups, J. Math. Soc. Japan 27 (1975), 600-612.

21. W. Thurston, The geometry and topology of 3-manifolds, Lecture notes, Princeton University, 1977.

22. B. Wehrfritz, Infinite Linear Groups, Spring, Berlin, 1973. 